\title{
Lab Marks-Cumulative Management System
}

\author{
Dayanand G Savakar ${ }^{1}$, Ravi Hosur ${ }^{2}$ \\ ${ }^{1}$ Rani Chennamma University, Belagavi \\ ${ }^{2}$ BLDEA's Vachana Pitamaha Dr. P.G.Halaktti College of Engg. \& Tech, Bijapur
}

\begin{abstract}
The system is designed to help the admin of the department to monitor the students of their regularity in the laboratory with their attendance report of the class. The system tracks the student with respect to the data given by the staff pertaining presence for their laboratory conducted, depending on which the system evaluates the performance of a student in conducting the experiment. It provides facilities to maintain the student reports obtained for every laboratory in the form of marks sheet. This project is going to help us to reduce the traditional paper-based data maintenance and verification/validation. This system will benefit for both the instructor and the staff. The system will help the staff to know the percentages of attendance and also track the records of previous years.
\end{abstract}

Keywords: Attendance, Laboratory, Paper, Records, System

\section{Introduction}

Attendance Management System is a web based application developed for managing student's attendance details using relational database. It facilitates to access the attendance information of a particular student in a particular laboratory. The information is sorted by the operators, which will be provided by the teacher for a particular class. This system will also help in evaluating attendance eligibility criteria of a student.

Main objective of this software is to provide software solution to colleges for managing student's attendance details in database. The purpose of developing this project is to update student attendance weekly/monthly. Comparing with existing system this method will help management team to easily analyze student's attendance details for weekly and monthly basis. In this the teachers engaging different laboratories are required to submit the attendance of the students present in their lab regularly.

The purpose of developing attendance management system is to computerized the tradition way of taking attendance. Another purpose for developing this software is to generate the report automatically at the end of the session or in the between of the session. An easier way to get to know the percentage of attendance in every laboratory. The system tries to reduce manual work, time and possibilities of mistakes, Reduces human efforts, Faster way to get detailed attendance, A paperless processing

\section{Review of Literature}

Staffs use to do all their tasks independently and after completion of the work, they integrate their work according to the requirement which is not efficient and takes more time. The existing system is based on manual work like maintaining record books. If any student attendance details are required, then one has to search through the number of records in register. This is more time consuming. Information will not be accessed easily. Manual calculation of percentage of student's attendance. In the existing system, student has to walk into specific teacher for information on attendance.
The method [1], witness's huge notice and a wealth of assure in content-based image recovery as a rising technology. It also a horizontal way for a huge number of new techniques and systems, get various new citizens include. In this piece, we survey almost 300 new hypothetical and experimental charity in the existing decade related to image recovery and regular image clarification. We also discuss significant challenges involved in the difference of existing image recovery techniques to build systems that can be useful in the genuine world. In retrospect of what has been achieved so far, we also work out what the prospect may hold for image recovery study.

Predictable methods [2] of image revival require that metadata is connected with the image, usually known as keywords. Though some content based image retrieval systems utilize together semantic and prehistoric attributes to relation search principle, history has proven that it is tricky to remove linguistic in sequence from a $2 \mathrm{D}$ picture. In this observe, activity theory is used as a foundation to express how semantic in sequence can be retrieved from objects recognized in a picture. Via an picture segmentation method.

By The Berkeley Digital Library Project, and merge it with, a high-level accepting of he picture can be established Content-Based Image Retrieval [3] has become one of the popular most research areas. Many diagram attribute representations contain been explored and many systems build. While, these research information found the foundation of satisfied based image recovery, the kindness of the future approaches is incomplete. Specially, these efforts have comparatively overlooked two different characteristics of systems the space between towering level concepts and low level skin texture bias of human compassion of visual content. Which electively takes into account the above two uniqueness in CBIR. During the recovery process, the user's high level query and insight partisanship are captured by dynamically updated weights based on the user's advice. The provisional results over more than 70,000 images show that the future approach greatly reduces the user's effort of composing a doubt and capture the user's in sequence. 


\section{International Journal of Science and Research (IJSR) \\ ISSN (Online): 2319-7064}

Index Copernicus Value (2013): 6.14 | Impact Factor (2015): 6.391

Application feedback [4] scheme based on support vector equipment have been generally used in content-based image retrieval. However, the arrangement of based application criticism is frequently abridged when the figure of labeled positive advice sample is little. This is mostly due to three reasons a classifier is disturbed on a little sized teaching locate, and over suitable happens since the number of characteristic dimensions is much senior than the size of the preparation set. In this document, we expand a device to overcome these troubles. To speak to the first two troubles, we propose an asymmetric container based. For the third problem, we combine the random subspace method and SVM for application feedback, which is named random subspace SVM (RS-SVM). Finally, by AB-SVM and RSSVM, an asymmetric bag and accidental subspace SVM (ABRS-SVM) is build to solve these three problems and further improve the application feedback performance. Some researchers used Image processing techniques for security[5][6] and for agriculture and horticulture produce[7][8].

\section{Proposed System}

The Online Attendance Management System provides the student information on their attendance. This project maintains all the information of student's attendance and provides flexible way of accessing the information. This project helps the teachers to fast their calculation on student attendance in less time.

- User Friendly: The proposed system is user friendly because the retrieval and storing of data is fast and data is maintained efficiently. Moreover the graphical user interface is provided in the proposed system, which provides user to deal with the system very easily

- Reports Are Easily Generated: Reports can be easily generated in the proposed system so user can generate the report as per the requirement (monthly) or in the middle of the session

- Very Less Paper Work: The proposed system requires very less paper work. All the data is stored into the computer immediately and reports can be generated through computers. Moreover work becomes very easy because there is no need to keep data on registers

- Computer Operator Control: Computer operator control will be there so no chance of errors. Moreover storing and retrieving of information is easy. So work can be done speedily and in time

The purpose of the Feasibility study is use to check whether a project is significance undertaking. The process is followed in making this determination is called a Feasibility Study. Keeping in a mind that the benefit of the organization, once it has been determined that the project is feasible, the analyst can decided and he can make prepare for project specification, that finalizes the project requests. For the period of study different tests of feasibility are studied.

Feasibility study is needed to project; the complete project it going to done from the feasibility study. For this all information and requirements is essentially needed. The initial requirement is the literature survey of the system. Even some of the software and hardware id needed to develop or build the system. By taking this all requirements the whole application can be developed.

System design is the process of defining the architecture, components, modules, interfaces, and data for a system to satisfy satisfied requirements.

The design phase can be broadly classified in two levels: Preliminary or high level design, Detailed design, The preliminary design can be further divided into two sub categories Function Oriented Software Design and Object Oriented Software Design

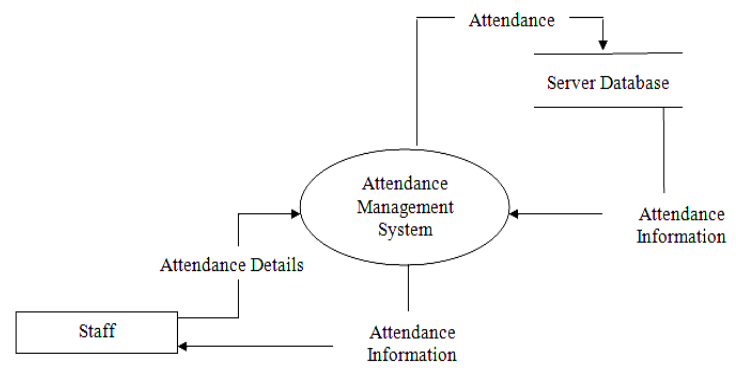

The context-level DFD is then exploded to produce a Level 1 DFD which models the details of the system. The Level 1 DFD shows how the system is divided into sub-systems (processes), and how each processes deals with one or more of the data flows to or from an external entity, and how the processes together provide all of the functionality of the system. The level 1 DFD also identifies the internal data stores which must be there for the system to do its job, and shows the data flow between the various parts of the system.

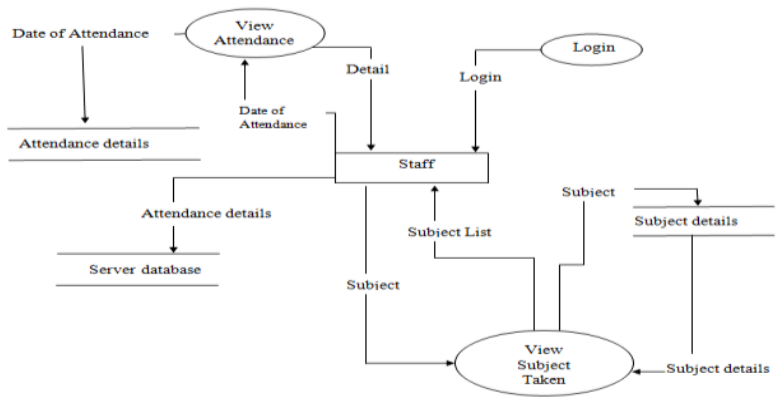

The Level 2 DFD is the further decomposition of Level 1 processes into sub-processes (sub-systems) which give detailed description of the data flow in each process. It shows that activities of the staff how they are managing the attendance report date-wise and all details are represented with the help of DFD. Here staff should login with the help of their userId and password. After login process, staff will get the details of his/her allotted subject list among those subject staff will select his allotted subject.

\section{Conclusion}

As the requirements are growing for the automation so we are introducing our application for the programmers to save their time in understanding the code. The system is simple by accepting the input and automatically generates comments for functional line of code. The system is developed using ASP.NET Using C\# fully meets the objectives of the system which it has been developed. The system has reached a steady state where all bugs have been 


\section{International Journal of Science and Research (IJSR) \\ ISSN (Online): 2319-7064}

Index Copernicus Value (2013): 6.14 | Impact Factor (2015): 6.391

eliminated. The system is operated at a high level of efficiency and all the teachers and user associated with the system understands its advantage. The system solves the problem. It was intended to solve as requirement specification.

\section{Future Enhancement}

The system can be further enhanced for several other functionalities that can be added at the college level for monitoring and recording the data for future usage. The database can be maintained for larger set of data that is dynamic.

\section{References}

[1] R. Datta, D. Joshi, and J.Z. Wang (2007), "Image Retrieval: Ideas, Influences, and Trends" ACM Computing Surveys, vol. 40, article 5

[2] A.W.M. Smeulders, M. Worring, S. Santini, A. Gupta, and R. Jain (2000), "Content-Based Image Retrieval," IEEE Trans. Pattern Analysis and Machine Intelligence, vol. 22, no. 12, pp. 1349-1380

[3] Y. Rui, T.S. Huang, M. Ortega, and S. Mehrotra(1998), "Relevance Feedback: A Power Tool for Interactive Content-Based Image Retrieval," IEEE Trans. Circuits and Systems for Video Technology, vol. 8, no. 5, pp. 644-655

[4] X.S. Zhou and T.S. Huang (2003), "Relevance Feedback in Image Retrieval: A Comprehensive Review," Multimedia Systems, vol. 8,pp. 536-544D.G.Savakar, Anand Ghuli (2015), "Digital Watermarking A Combined Approach by DWT, Chirp-Z and Fast WalshHadamard Transform", IJCTA, Vol. 5 No.6, pp 20062010.

[5] D.G.Savakar, Anand Ghuli (2015), "Digital Watermarking as a distributed noise by Discrete Wavelet Transformation, Fast Fourier Transformation and Fast Walsh-Hadamard Transform to study the sensitivity between Robustness and Fidelity", IJCA, Issue 1, Volume 5, pp 102-107

[6] Dayanand G. Savakar (2012), Identification and Classification of Bulk Fruits Images using Artificial Neural Networks. International Journal of Engineering and Innovative Technology (IJEIT), Volume 1, Issue 3, Pages: $35-40$

[7] Dayanand G. Savakar (2012), Recognition and Classification of Similar Looking Food Grain Images using ANN, Journal of Applied Computer Science and Mathematics ,Volume 13(6), Pages: 61- 65 